\title{
Particle and Nuclear Physics Aspects of the Big Bang Nucleosynthesis
}

\author{
Toshitaka Kajino* \\ National Astronomical Observatory of Japan, Osawa 2-21-1, Mitaka, Tokyo 181-8588, Japan \\ Department of Astronomy, The University of Tokyo, 7-3-1 Hongo, Bunkyo-ku, Tokyo 113-0033, \\ Japan, E-mail: kajino@nao.ac.jp \\ Motohiko Kusakabe \\ School of Liberal Arts and Science, Korea Aerospace University, Goyang-city, Gyeonggi-do \\ 412-791, Korea
}

\section{A. Baha Balantekin}

Department of Physics, University of Wisconsin, Madison, WI 53706, USA

\section{Yamac Pehlivan}

Department of Physics, Mimar Sinan Fine Arts University, Sisli, Istanbul 34380, Turkey

\section{Grant J. Mathews}

Center for Astrophysics, Department of Physics, University of Notre Dame, Notre Dame, IN 46556, USA

\section{Myung-Ki Cheoun}

Department of Physics, Soongsil University, Seoul 156-743, Korea

The Big Bang Nucleosynthesis is the pillars of modern cosmology. It has recently been suggested theoretically that axions which are candidate for dark matter particles in the standard particle theory could condensate between the epochs of nucleosynthesis and last photon scattering in the early universe, which would result in cooling of photons with the shift of baryon-to-photon ratio between the two epochs. This renders a solution to the overproduction problem of primordial ${ }^{7} \mathrm{Li}$ abundance. However, there arises another serious difficulty of overpredicting primordial deuterium abundance. We propose a hybrid dark matter model with both axions and relic supersymmetric particles to solve these overproduction problems of the primordial deuterium and ${ }^{7} \mathrm{Li}$ abundances simultaneously. We also review several different cosmological models for the Big Bang Nucleosynthesis.

XII International Symposium on Nuclei in the Cosmos

August 5-12, 2012

Cairns, Australia

\footnotetext{
${ }^{*}$ Speaker.
} 


\section{Introduction}

One of the biggest questions in modern cosmology is why the universe is most likely flat $\Omega_{\mathrm{tot}}=1$ and how its expansion is accelerating. Although the standard cosmology assuming dark energy $\Omega_{\Lambda}=0.714$, dark matter $\Omega_{\mathrm{CDM}}=0.240$, and baryonic matter $\Omega_{\mathrm{b}}=0.046$ 《1] fulfills these requirements, real scientific challenge is to solve the unknown nature of these cosmological parameters, based on the precise knowledge of particle and nuclear physics. The $\Omega_{\mathrm{i}}$-values, Hubble constant $\mathrm{H}_{0}$, and other cosmological parameters are constrained from the combined fits of the observed magnitude-redshift relation of Type Ia supernovae, cosmic microwave background (CMB) anisotropies, and lensing effects of galaxy clusters, etc. The $\Omega_{\mathrm{b}}$-value is independently determined from the Big Bang Nucleosynthesis (BBN). The BBN is also the pillars to understand the elementary and nuclear processes in the early universe.

Recent observations of metal-deficient halo stars using high-dispersion spectrograph have indicated a finite ${ }^{7} \mathrm{Li}$ abundance, suggesting the primordial origin [2]. However, the amount of ${ }^{7} \mathrm{Li}$ needed to be consistent with the CMB observations [3] is significantly more than ${ }^{7} \mathrm{Li}$ observed in old halo stars [4]. It is also suggested observationally that ${ }^{6} \mathrm{Li}$ shows a plateau-like abundance in some metal-deficient halo stars [4]. The abundance level is about $\sim 5 \%$ of ${ }^{7} \mathrm{Li}$, whereas the cosmological theory of BBN predicts negligible amounts of ${ }^{6} \mathrm{Li}$ compared to the detected level.

Even though both ${ }^{7} \mathrm{Li}$ and ${ }^{6} \mathrm{Li}$ can be produced and destroyed in stars, old halo dwarf stars are thought to have gone through little nuclear processing [5]. Recent improvements in the observational and experimental data seem to make the discrepancy worse [6, 7]. One possible solution is to invoke either nuclear physics hitherto excluded from the BBN [8, 9] or new physics such as variations of fundamental coupling constants [10,11, and particles not included in the Standard Model. Effects of massive neutral relic particles on BBN were also extensively studied in [12] and references therein. We here discuss particle and nuclear physics aspects of the BBN, focussing on the discussions how to solve these lithium problems.

\section{Primordial Abundances}

In the standard cosmology only the lightest elements $\mathrm{D},{ }^{3} \mathrm{He},{ }^{4} \mathrm{He},{ }^{6} \mathrm{Li}$ and ${ }^{7} \mathrm{Li}$ are presumably produced in the hot Big Bang expansion of the early universe, although cosmological theory of the inhomogeneous BBN [13, 14] and other non-standard models predict production of the intermediate-to-heavy mass elements like ${ }^{9} \mathrm{Be}$ and ${ }^{10,11} \mathrm{~B}$ as well as the lightest elements.

For the primordial $\mathrm{D}$ abundance, the mean value estimated from Lyman- $\alpha$ absorption systems in the foreground of high redshift quasi-stellar objects is $\log (\mathrm{D} / \mathrm{H})=-4.55 \pm 0.03$ [15]. We adopt this value together with a $2 \sigma$ uncertainty, i.e., $2.45 \times 10^{-5}<\mathrm{D} / \mathrm{H}<3.24 \times 10^{-5}$. ${ }^{3} \mathrm{He}$ abundance measurements in Galactic HII regions through the $8.665 \mathrm{GHz}$ hyperfine transition of ${ }^{3} \mathrm{He}^{+}$yield a value of ${ }^{3} \mathrm{He} / \mathrm{H}=(1.9 \pm 0.6) \times 10^{-5}$ [16]. Although the constraint should be rather weak considering its uncertainty, we take a $2 \sigma$ upper limit and adopt ${ }^{3} \mathrm{He} / \mathrm{H}<3.1 \times 10^{-5}$. We also utilize a limit on the sum of primordial abundances of $\mathrm{D}$ and ${ }^{3} \mathrm{He}$ taken from an abundance for the protosolar cloud determined from observations of solar wind, i.e., $\left(\mathrm{D}+{ }^{3} \mathrm{He}\right) / \mathrm{H}=(3.6 \pm 0.5) \times 10^{-5}$ [17]. This abundance can be regarded as constant at least within the standard cosmology since it is not affected by stellar activities significantly despite an effect of D burning into ${ }^{3} \mathrm{He}$ via ${ }^{2} \mathrm{H}(p, \gamma){ }^{3} \mathrm{He}$ 
would exist [18]. For the primordial ${ }^{4} \mathrm{He}$ abundance, we adopt two different constraints from recent reports: $Y_{\mathrm{p}}=0.2565 \pm 0.0051$ [19] and $Y_{\mathrm{p}}=0.2561 \pm 0.0108$ [20] both of which are derived from observations of metal-poor extragalactic HII regions. Adding $2 \sigma$ uncertainties leads to $0.2463<$ $Y_{\mathrm{p}}<0.2667$ [19] and $0.2345<Y_{\mathrm{p}}<0.2777$ [20]. ${ }^{6} \mathrm{Li}$ abundance of metal-poor halo stars (MPHSs), yields the upper limit of ${ }^{6} \mathrm{Li} / \mathrm{H}=(7.1 \pm 0.7) \times 10^{-12}$ [4]. Adding a $2 \sigma$ uncertainty, we adopt ${ }^{6} \mathrm{Li} / \mathrm{H}<8.5 \times 10^{-12}$. For the ${ }^{7} \mathrm{Li}$ abundance, we adopt the limits $\log \left({ }^{7} \mathrm{Li} / \mathrm{H}\right)=-12+(2.199 \pm$ 0.086) (with $95 \%$ C. L.) derived from recent observations of MPHSs in the 3D nonlocal thermal equilibrium model [21], i.e. $1.06 \times 10^{-10}<{ }^{7} \mathrm{Li} / \mathrm{H}<2.35 \times 10^{-10}$.

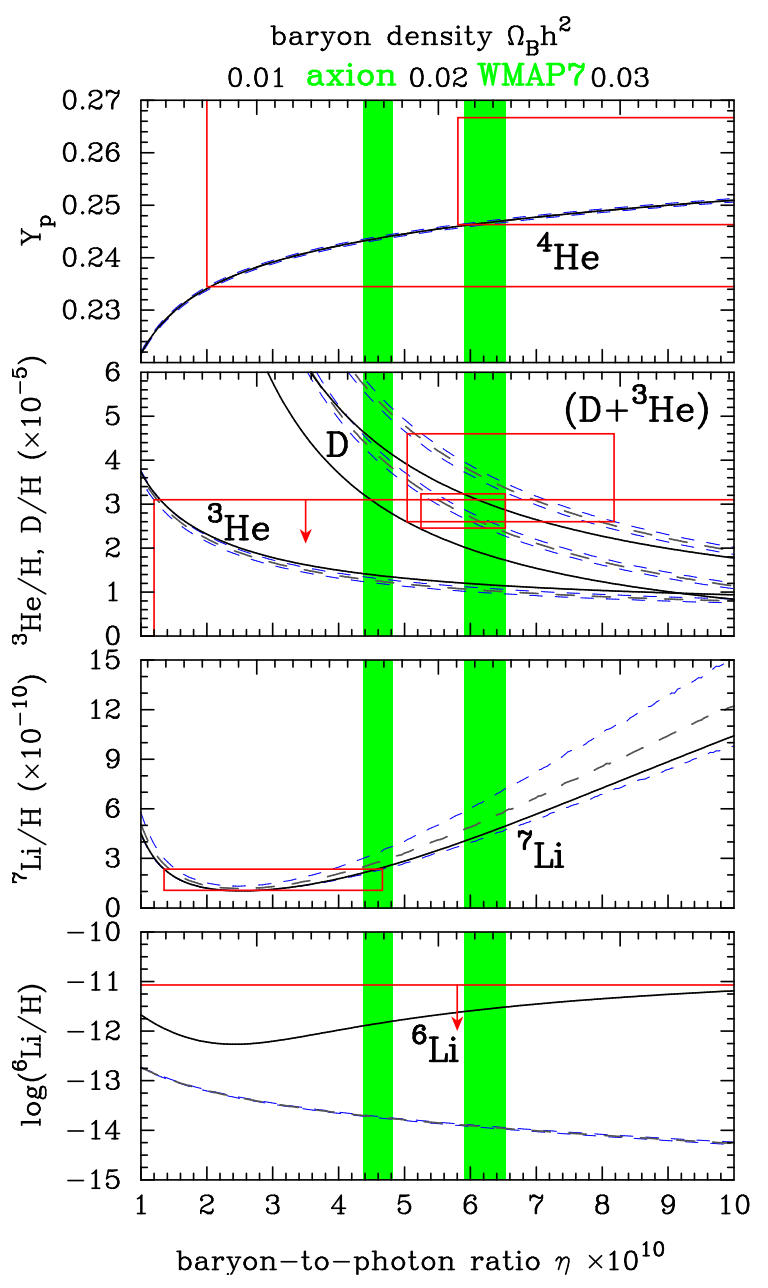

Figure 1: Abundances of ${ }^{4} \mathrm{He}$ (mass fraction), $\mathrm{D},{ }^{3} \mathrm{He},{ }^{7} \mathrm{Li}$ and ${ }^{6} \mathrm{Li}$ (number ratio relative to $\mathrm{H}$ ) as a function of the baryon-to-photon ratio $\eta$ or the baryon energy density parameter $\Omega_{B} h^{2}$ of the universe. The thick dashed curves are for Standard BBN (SBBN). The thin dashed curves around them show the regions of $95 \%$ C. L. in accordance with the nuclear reaction rate uncertainties. The boxes correspond to the adopted abundance constraints on the SBBN model. The vertical stripes represent the $2 \sigma$ limits on $\Omega_{B} h^{2}$ or $\eta$ for the SBBN model (taken from the constraint by CMB-WMAP [37] and labeled as WMAP7) and for the axion BEC model (labeled as axion). The solid curves are the results obtained with our hybrid model of the long-lived decaying particle model with parameters fixed to $\left(\tau_{X}, \zeta_{X}\right)=\left(10^{6} \mathrm{~s}, 2 \times 10^{-10} \mathrm{GeV}\right)$ (see text) [12]. 


\section{Big Bang Nucleosynthesis}

We carried out Standard BBN network calculations using Kawano's code [22, 23] by including Sarkar's correction for ${ }^{4} \mathrm{He}$ abundances [24]. We checked our code against the publicly available code PArthENoPE 1.0 [25] with their uncertainties being included [26], and found that the differences in $\mathrm{D},{ }^{3} \mathrm{He}$, and ${ }^{7} \mathrm{Li}$ abundances between two codes are less than $0.8 \%$, and that for ${ }^{4} \mathrm{He}$ is less than $0.2 \%$. JINA REACLIB Database V1.0 [27] is used for light nuclear $(A \leq 10)$ reaction rates including uncertainties together with data [28, 29, 30]. Adopted neutron lifetime is $878.5 \pm 0.7_{\text {stat }} \pm 0.3_{\text {sys }} \mathrm{s}$ [31] based on improved measurements [32]. Taking into account the uncertainties in these rates [27], we employ regions of 95\% C. L. in our calculations.

\subsection{Axion Dark Matter Model}

Recently a model to solve the ${ }^{7} \mathrm{Li}$ problem was proposed [33, 34]. It was suggested that dark matter axions could form a Bose-Einstein condensate (BEC). Such a condensate would cool the photons between the end of BBN and epoch of photon decoupling, reducing the baryon-tophoton ratio that the CMB-WMAP data analysis infers, as compared to its BBN value [35]. An alternative mechanism for such a cooling is resonant oscillations between photons and light abelian gauge bosons in the hidden sector [36]. There are two prima facie problems with the axion BECphoton cooling hypothesis: it overpredicts primordial deuterium (D) abundance as well as the effective number of neutrinos. Even though D is easy to destroy, one does not expect the sum of abundances of D and ${ }^{3} \mathrm{He}$ to change significantly in the course of cosmic evolution [18]. Hence it is important to find a parameter region in which predicted abundances of $\mathrm{D}$ and ${ }^{7} \mathrm{Li}$ are consistent with observations.

Figure 1 shows the abundances of ${ }^{4} \mathrm{He}\left(Y_{\mathrm{p}}\right.$; mass fraction), $\mathrm{D},{ }^{3} \mathrm{He},{ }^{7} \mathrm{Li}$ and ${ }^{6} \mathrm{Li}$ (number ratio relative to $\mathrm{H}$ ) as a function of the baryon-to-photon ratio $\eta$ or the baryon energy density parameter $\Omega_{B} h^{2}$ of the universe, where $h$ is the Hubble constant in units of $100 \mathrm{~km} / \mathrm{s} / \mathrm{Mpc}$. The thick dashed curves are the results of the Standard BBN (SBBN) with a neutron lifetime of $878.5 \pm 0.8 \mathrm{~s}$. Thin dashed curves around them show regions of $95 \%$ C. L. from uncertainties in the nuclear reaction rates. The boxes represent adopted abundance constraints as summarized above. The vertical stripes correspond to the $2 \sigma$ limits on $\Omega_{B} h^{2}$ or $\eta$. The values provided by WMAP [37] (labeled WMAP7) are

$$
\Omega_{B} h^{2}=0.02258_{-0.00112}^{+0.00114} \quad \eta=\left(6.225_{-0.309}^{+0.314}\right) \times 10^{-10} .
$$

Values predicted by the BEC model (labeled axion) are smaller by a factor of $(2 / 3)^{3 / 4}$ at the BBN epoch [35]:

$$
\Omega_{B} h^{2}=0.01666_{-0.00083}^{+0.00084} \quad \eta=\left(4.593_{-0.228}^{+0.232}\right) \times 10^{-10} .
$$

It can be seen that the adoption of the $\eta$ value from WMAP leads to a ${ }^{7} \mathrm{Li}$ abundance calculated in the axion BEC model, which is in reasonable agreement with the observations. However, we lose the important consistency in D abundance. Ref. [35] noted that astronomical measurements of primordial D abundance can have a significant uncertainty as well as a possibility that $\mathrm{D}$ is burned by nonstandard stellar processes. Even if their assumption were true, stellar processes are not expected to change the sum of $\mathrm{D}$ and ${ }^{3} \mathrm{He}$ abundances [18]. As seen in Fig. 11 the constraint on $\left(\mathrm{D}+{ }^{3} \mathrm{He}\right) / \mathrm{H}$ abundance seems to exclude the original axion BEC model. Ultimately, this model is viable only when the abundance of $\left(\mathrm{D}+{ }^{3} \mathrm{He}\right) / \mathrm{H}$ is reduced through some exotic processes. 


\subsection{Hybrid Dark Matter Model: Axions and Neutral Relic Particles}

It is known that nonthermal photons can be generated through electromagnetic energy injections by the radiative decay of long-lived particles after the BBN epoch [38, 39]. Long-lived particles which radiatively decay are motivated by physics beyond the standard model like supersymmetry (SUSY). Candidates of such particles include a neutralino decaying to gravitino through gravitational interaction and others [40]. These nonthermal photons can photodisintegrate background light elements [38, 41, 42, 43, 44, 45]. We adopt the method of Ref. [45] to calculate the nonthermal nucleosynthesis, where we incorporated new thermal reaction rates as described above. In addition, we adopt updated reaction rates of ${ }^{4} \mathrm{He}$ photodisintegration [46] derived from the cross section data using precise measurements with laser-Compton photons [47, 48]. ${ }^{4} \mathrm{He}$ destruction by photons and nuclei in thermal bath is already included in our calculation. Effects of electromagnetic energy injection depend on two parameters. One is $\zeta_{X}=\left(n_{X}^{0} / n_{\gamma}^{0}\right) E_{\gamma 0}$ where $\left(n_{X}^{0} / n_{\gamma}^{0}\right)$ is the number ratio of the decaying particle $X$ and the background radiation before the decay of $X$, and $E_{\gamma 0}$ is the energy of photon emitted at the radiative decay. The other is $\tau_{X}$, the lifetime of the $X$ particle.

Figure 2 shows the parameter space in our hybrid model [12]. For ${ }^{4} \mathrm{He}$, we adopt the conservative constraint with larger uncertainty [20]. We also show the contour for ${ }^{6} \mathrm{Li}$ production at the observed level, i.e., ${ }^{6} \mathrm{Li} / \mathrm{H}=7.1 \times 10^{-12}$. This figure shows the result of nonthermal nucleosynthesis induced by the radiative decay of long-lived particles with the $\eta$ value of the axion BEC model [Eq. [3.2] ]. Except for $\mathrm{D}$ and ${ }^{7} \mathrm{Li}$, contours are similar to those represented in Ref [45] where BBN epoch $\eta$ value is assumed to be the same as WMAP $\eta$ value [Eq. 3.11].

In the very small colored region, calculated primordial abundances of all nuclides including $\mathrm{D}$ and ${ }^{7} \mathrm{Li}$ are simultaneously in ranges of adopted observational constraints. We conclude that the present model eliminates the main drawback of the original axion BEC model by reducing primordial D abundance via ${ }^{2} \mathrm{H}(\gamma, n){ }^{1} \mathrm{H}$ reaction, where $\gamma^{\prime}$ 's are nonthermal photons. We note that the decaying particle model with the WMAP $\eta$ value cannot resolve the ${ }^{7} \mathrm{Li}$ problem by itself (see Fig. 1) 45, 49.

The effect of the radiative decay on other elemental abundances is not significant except for ${ }^{7} \mathrm{Li}$ and D. Since energetic photons produced quickly collide with background photons and create $e^{+} e^{-}$pairs, nonthermal photon spectra developed by the decay has a cutoff energy $E_{\mathrm{C}}=m_{e}^{2} / 22 T$ where $m_{e}$ is the electron mass [39]. The decay at earlier (hotter) universe then triggers nonthermal photons with lower cutoff energies. The threshold energies of ${ }^{7} \mathrm{Be}$ and $\mathrm{D}$ photodisintegration, ${ }^{7} \mathrm{Be}+\gamma \rightarrow{ }^{3} \mathrm{He}+{ }^{4} \mathrm{He}$ and $\mathrm{D}+\gamma \rightarrow n+p$ are $1.5866 \mathrm{MeV}$ and $2.2246 \mathrm{MeV}$, respectively. These two nuclei are very fragile against photodisintegration. When the decay occurs early at relatively high $T_{9} \equiv T /\left(10^{9} \mathrm{~K}\right) \gtrsim 10^{-2}$ which corresponds to $\tau_{X} \lesssim 10^{6} \mathrm{~s}$, nonthermal photon spectra contain photons to dissociate ${ }^{7} \mathrm{Be}$ and $\mathrm{D}$, while keeping other nuclides intact. The gray region indicates parameters which result in moderate destruction of $\mathrm{D}$ which is overproduced in the original BEC model because of low $\eta$. Above that region, $\mathrm{D}$ is destroyed too much by photodisintegration, while below it $\mathrm{D}$ abundance is too high.

We carry out the BBN calculation in our hybrid model with a fixed set of parameters given by $\left(\tau_{X}, \zeta_{X}\right)=\left(10^{6} \mathrm{~s}, 2 \times 10^{-10} \mathrm{GeV}\right)$ and the $\eta$ value given in Eq. (3.2). This corresponds to the point indicated with a star in Fig. (2). At $0.06 \gtrsim T_{9} \gtrsim 7 \times 10^{-3}$ (corresponding to the cosmic time of $t \sim$ 


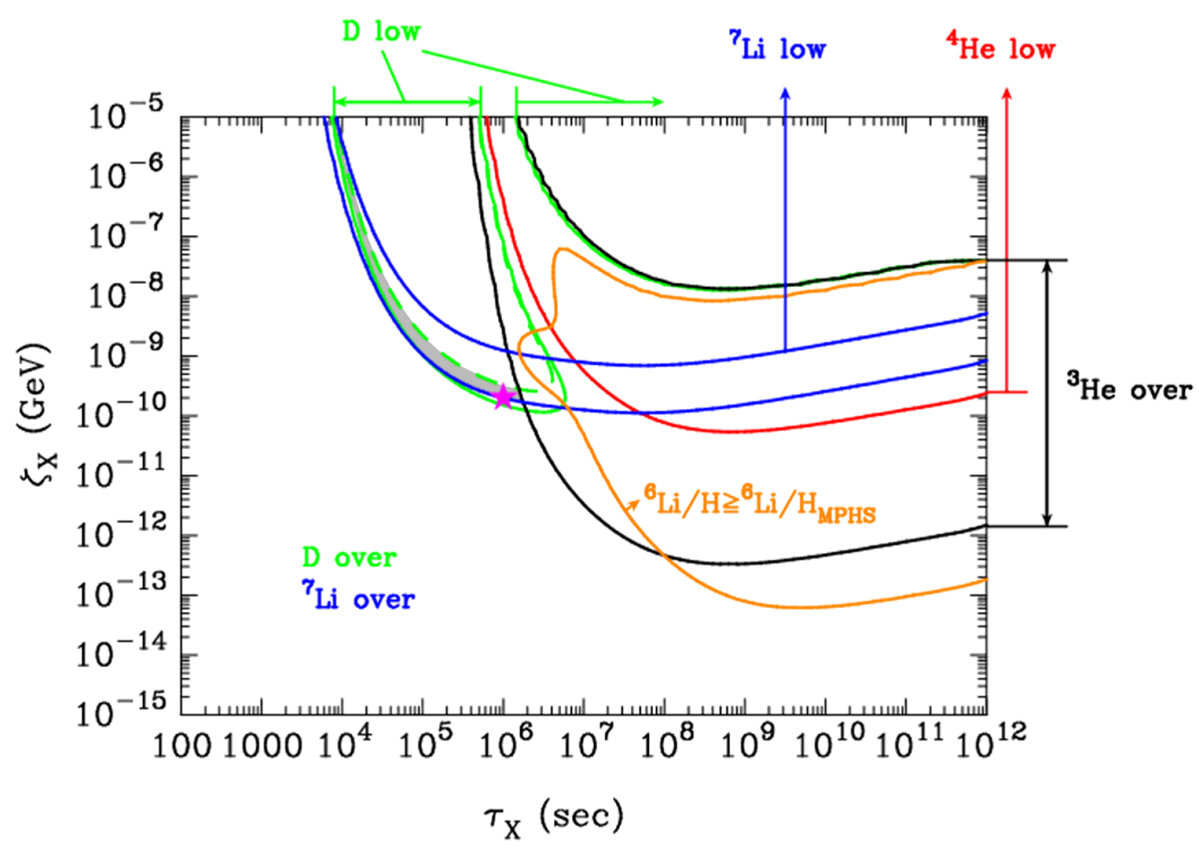

Figure 2: Parameter space of the hybrid model $\left(\tau_{X}, \zeta_{X}\right)$ for the value of $\eta=4.6 \times 10^{-10}$ provided by the axion BEC model. The contours identify the regions where the nuclei are overproduced or underproduced ("over" and "low", respectively) with respect to the adopted abundance constraints. ${ }^{4} \mathrm{He}$ mass fraction (red line) and ${ }^{3} \mathrm{He} / \mathrm{H}$ (black lines), D/H (green solid and dashed lines for upper and lower limits, respectively), and ${ }^{7} \mathrm{Li} / \mathrm{H}$ (blue line) number ratios are shown. The orange line is the contour of ${ }^{6} \mathrm{Li} / \mathrm{H}=7.1 \times 10^{-12}$. In the gray-colored region all abundances are within the limits of observational constraints [12].

$\left.5 \times 10^{4}-4 \times 10^{6} \mathrm{~s}\right)$, the ${ }^{2} \mathrm{H}(\gamma, n)^{1} \mathrm{H}$ reaction induced by non-thermal photons destroy $\mathrm{D}$ and increase $n$ abundances. We find a slight decrease in ${ }^{7} \mathrm{Be}$ abundance. This is caused through reactions ${ }^{7} \mathrm{Be}\left(\gamma,{ }^{3} \mathrm{He}\right){ }^{4} \mathrm{He}$ (threshold energy: $\left.1.5866 \mathrm{MeV}\right),{ }^{7} \mathrm{Be}(\gamma, p){ }^{6} \mathrm{Li}(5.6858 \mathrm{MeV})$, and ${ }^{7} \mathrm{Be}(\gamma, 2 p n){ }^{4} \mathrm{He}$ $(9.3047 \mathrm{MeV})$. The second reaction increases the ${ }^{6} \mathrm{Li}$ abundance. Finally, at $T_{9} \lesssim 7 \times 10^{-3}$, where the abundance of long-lived $X$ particle is already less than $3 \%$ of the initial abundance, effect of ${ }^{4} \mathrm{He}$ photodisintegration is to increase ${ }^{3} \mathrm{H}$ and $n$ abundances.

As noted above in Fig. 1, D abundance disagrees with observation significantly in Axion BEC model. The ${ }^{7} \mathrm{Li}$ abundance is slightly larger than the observational constraint. However, since the ${ }^{7} \mathrm{Li}$ abundance is affected significantly by uncertainties in nuclear reaction rates, it is marginally allowed (see Fig. 1 and also Fig. 1 of Ref. [35]). In BBN model with long-lived particle, ${ }^{7} \mathrm{Li}$ abundance is larger than the observation. In our hybrid model [12], abundances of all nuclides are consistent with observations.

For the hybrid model with this parameter set, the final abundance of $\left({ }^{7} \mathrm{Li}+{ }^{7} \mathrm{Be}\right) / \mathrm{H}$ is smaller than that in $\operatorname{SBBN}\left(\eta=6.2 \times 10^{-10}[37)\right.$ by $2.9 \times 10^{-10}$. The abundance is reduced from the small $\eta$ value by $2.5 \times 10^{-10}$, and further reduced from ${ }^{7} \mathrm{Be}$ photodisintegration triggered by the radiative decay of long-lived particle by $0.4 \times 10^{-10}$. The photon cooling by axion is thus the main reason of small ${ }^{7} \mathrm{Li}$ abundance. This study teaches us the following lesson: It is impossible to reduce ${ }^{7} \mathrm{Li}$ abundance without reducing D abundance too much in the BBN model without photon cooling. 
However, when D is overabundant as in the BBN model with photon cooling, the radiatively decaying particle can achieve a significant $\mathrm{D}$ destruction associated with a subdominant ${ }^{7} \mathrm{Li}$ reduction [12].

In Fig. 1] solid lines show the results for the SBBN + long-lived decaying particle model with the same parameter values, i.e., $\left(\tau_{X}, \zeta_{X}\right)=\left(10^{6} \mathrm{~s}, 2 \times 10^{-10} \mathrm{GeV}\right)$. Obviously the abundances of $\mathrm{D}$ and ${ }^{7} \mathrm{Li}$ (produced partly as ${ }^{7} \mathrm{Be}$ ) are reduced, while that of ${ }^{6} \mathrm{Li}$ is increased from those of SBBN.

\section{Alternative Model}

\subsection{Dark Matter Model of Charged Relic Particles}

If a relic charged particle $\left(X^{-}\right)$like stau, which is a super-partner of tau-lepton in supersymmetry (SUSY) and has a long life, existed in $\mathrm{BBN}$ epoch, it binds to ${ }^{7} \mathrm{Be}$ to form ${ }^{7} \mathrm{Be}_{X}$ which is destroyed by proton capture reaction through both atomic [50] and nuclear [51] excited state of ${ }^{8} \mathrm{~B}_{X}$. This scenario provides one solution to the lithium problem since a ${ }^{7} \mathrm{Be}$ destruction in BBN epoch leads to an overall reduction of primordial ${ }^{7} \mathrm{Li}$ abundance. However, model parameters, i.e., the lifetime and abundance of $X^{-}$, must be fine-tuned in order to escape from an overproduction of ${ }^{6} \mathrm{Li} \mathrm{[52]}$. The abundance of $X^{-}$must be higher than that of baryon (e.g. Refs. [53, 54, 55, 56]). Such a high abundance is, however, referred with caution [52].

\subsection{Time Dependent Coupling Constant}

We studied the constraints from primordial nucleosynthesis on a possible time-dependent strong coupling constant [10, 11]. This time-dependence is equivalent to the time-dependent quark masses. The present study incorporates updated light-element abundances and uncertainties along with a new independent analysis of the influence of such quark-mass variations on the resonance properties of the important ${ }^{3} \mathrm{He}(d, p){ }^{4} \mathrm{He}$ and ${ }^{3} \mathrm{H}(d, n){ }^{4} \mathrm{He}$ reactions. We find that the updated abundance and resonance constraints imply a narrower range on the possible quark-mass variations in the early universe[11]. We also find that, contrary to previous investigations, the optimum concordance region reduces to a (95\% C.L.) value of $-0.005 \lesssim \delta m_{q} / m_{q} \lesssim 0.01$ consistent with no variation in the averaged quark mass. We cannot resolve the ${ }^{7} \mathrm{Li}$ overproduction problem.

\subsection{Baryon Inhomogeneous Model}

We studied primordial nucleosynthesis in the neutron and proton segregated environments produced by baryon-number inhomogeneous big bang model. We found that there are several observational signatures of such neutron-rich and proton-rich BBN in the intermediate [13, 14] and heavy [57] mass nuclei. We carefully analyzed the allowed parameters for the inhomogeneous BBN in light of the CMB-WMAP constraints on the baryon-to-photon ratio and precise measurement which set the neutron lifetime to be $878.5 \pm 0.7 \pm 0.3 \mathrm{sec}$ [58]. The new lifetime reduces the mass fraction of ${ }^{4} \mathrm{He}$ by 0.0015 but does not significantly change the abundances of other isotopes. This enlarges the region of baryon-to-photon ratio constrained from $\mathrm{D}$ and ${ }^{4} \mathrm{He}$. The ${ }^{7} \mathrm{Li}$ abundance can be brought into concordance with observed $\mathrm{D}$ and ${ }^{4} \mathrm{He}$ abundances by using depletion factors as high as 9.3. The CMB-WMAP constraints, however, severely limit the allowed comoving ( $T=100$ GK) mean separation distance scale between inhomogeneous baryon-number density fluctuations to be $d_{i n h}=(1.3-2.6) 10^{5} \mathrm{~cm}$. 


\subsection{Cosmology with Extra Dimensions}

\subsubsection{Brane World Cosmology}

Cosmology with extra dimensions is of considerable interest as a step towards unification of elementary particles and fields. Randall and Sundrum [59] have shown that mismatch between of the scales of particle physics and gravity (hierarchy problem) can be solved by introducing noncompact extra dimensions, i.e. the brane world cosmology. In this model our universe is a submanifold embedded in a higher-dimensional spacetime. The Friedmann equation is modified by the appearance of extra term, dark radiation, which diminishes with cosmic scale factor as $a^{-4}$.

We showed that a wide range of negative dark radiation term is allowed [60] so that it reconciles the tension between the observed primordial ${ }^{4} \mathrm{He}$ and $\mathrm{D}$ abundances. The BBN constraints allow dark radiation between $-123 \%$ and $+11 \%$ of the background photon energy density at the epoch before the nucleosynthesis and the $e^{+} e^{-}$annihilation. Combining this with the constraint on the $\mathrm{CMB}$ anisotropies, we still find a wider range of negative dark radiation term. However, we cannot resolve the ${ }^{7} \mathrm{Li}$ overproduction problem.

\subsubsection{Disappearing Dark Matter Model and $\Lambda=0$ Cosmology}

It is an unavoidable consequence of the brane world cosmology that massive particles are metastable and can disappear from our universe (i.e. brane) into the 5-th bulk dimension 61. A massive dark matter particle (e.g. the lightest supersymmetric particle) is likely to have the shortest lifetime for disappearing into the bulk. We studied consistency between this new paradigm of disappearing dark matter and all cosmological constraints from the BBN of the light elements (except for lithium), the Type Ia supernovae at the highest redshift, the mass-to-light ratios of galaxy clusters with redshift, the fraction of X-ray emitting gas in rich clusters, and the CMB anisotropies [62]. A best $2 \sigma$ concordance region is identified corresponding to a mean lifetime for dark matter disappearance of $15 \leq \Gamma^{-1} \leq 80 \mathrm{Gyr}$.

We also explored observational constraints on a brane world cosmology in which the 5-th bulk dimensional is not empty. Allowing exchange of mass-energy between the bulk and the bane, evolution of matter fields to an observer on the brane is modified due to new terms in the energy momentum tensor describing this exchange. We studied the constraints from various cosmological observations on the flow of matter from the bulk into the brane, and found that a $\Lambda=0$ cosmology to an observer is possible very interestingly [63]. In this cosmology the observed cosmic acceleration is attributable to the flow of matter from the bulk to the brane. This model even accounts for the observed suppression of the CMB power spectrum at low multipoles.

\section{Conclusions}

We proposed a hybrid axion and massive relic particle model in which axions cool the photons and relic particles produce non-thermal photons to eliminate the overproduced $\mathrm{D}$ abundance in the original axion BEC model. Our hybrid model also produces ${ }^{6} \mathrm{Li}$ keeping ${ }^{7} \mathrm{Li}$ abundance at the level of Population II Spite plateau. Our work thus demonstrates that the ${ }^{7} \mathrm{Li}$ abundance can be consistent with observations without destroying the important concordance of light element abundances. 


\section{Acknowledgments}

This work was supported by Grants-in-Aid for Scientific Research of JSPS (200244035), JSPS Grant No.21.6817, U.S. National Science Foundation Grant No. PHY-0855082 and PHY-1205024, the Council of Higher Education of Turkey, and the University of Wisconsin Research Committee with funds granted by the Wisconsin Alumni Research Foundation.

\section{References}

[1] G. Hinshaw et al., arXiv:1212.5226 (2012).

[2] F. Spite and M. Spite, Astron. Astrophys. D 115, 357 (1982).

[3] E. Komatsu et al. [WMAP Collaboration], Astrophys. J. Suppl. 192, 18 (2011).

[4] M. Asplund et al., Astrophys. J. 644, 229 (2006).

[5] A. J. Korn et al., Nature 442, 657 (2006).

[6] R. H. Cyburt, B. D. Fields and K. A. Olive, JCAP 0811, 012 (2008).

[7] A. Coc et al., Astrophys. J. 744, 158 (2012).

[8] N. Chakraborty, B. D. Fields and K. A. Olive, Phys. Rev. D 83, 063006 (2011).

[9] R. H. Cyburt and M. Pospelov, Int. J. Mod. Phys. E 21, 1250004 (2012).

[10] A. Coc et al., Phys. Rev. D 76, 023511 (2007).

[11] M. K. Cheoun, T. Kajino, M. Kusakabe and G. J. Mathews, Phys. Rev. D 84, 043001 (2011).

[12] M. Kusakabe, A. B. Balantekin, T. Kajino and Y. Pehlivan, Phys. Lett. B 718, 704 (2013).

[13] R. N. Boyd and T. Kajino, Astrophys. J. 336 (1989), L55-L58.

[14] T. Kajino and R. N. Boyd, Astrophys. J. 359 (1990), 267-276.

[15] M. Pettini et al., Mon. Not. R. Astron. Soc. 391, 1499 (2008).

[16] T. M. Bania, R. T. Rood and D. S. Balser, Nature 415, 54 (2002).

[17] J. Geiss and G. Gloeckler, Space Sci. Rev. 84, 239 (1998).

[18] G. Steigman and M. Tosi, Astrophys. J. 453, 173 (1995); F. Iocco et al., Phys. Rept. 472, 1 (2009).

[19] Y. I. Izotov and T. X. Thuan, Astrophys. J. 710, L67 (2010).

[20] E. Aver, K. A. Olive and E. D. Skillman, JCAP 1005, 003 (2010).

[21] L. Sbordone et al., Astron. Astrophys. 522, A26 (2010).

[22] L. Kawano, NASA STI/Recon Technical Report N 92, 25163 (1992).

[23] M. S. Smith, L. H. Kawano and R. A. Malaney, Astrophys. J. Suppl. 85, 219 (1993).

[24] S. Sarkar, Rept. Prog. Phys. 59, 1493 (1996).

[25] O. Pisanti et al., Comput. Phys. Commun. 178, 956 (2008).

[26] P. D. Serpico et al., JCAP 0412, 010 (2004).

[27] R. H. Cyburt et al., Astrophys. J. Suppl. Ser. 189, 240 (2010). 
[28] P. Descouvemont et al., At. Data Nucl. Data Tables 88, 203 (2004).

[29] S. Ando, R. H. Cyburt, S. W. Hong and C. H. Hyun, Phys. Rev. C 74, 025809 (2006).

[30] R. H. Cyburt and B. Davids, Phys. Rev. C 78, 064614 (2008).

[31] A. P. Serebrov and A. K. Fomin, Phys. Rev. C 82, 035501 (2010).

[32] A. P. Serebrov et al., Phys. Lett. B 605, 72 (2005).

[33] P. Sikivie and Q. Yang, Phys. Rev. Lett. 103, 111301 (2009).

[34] O. Erken, P. Sikivie, H. Tam and Q. Yang, arXiv:1111.1157 [astro-ph.CO].

[35] O. Erken, P. Sikivie, H. Tam and Q. Yang, Phys. Rev. Lett. 108, 061304 (2012).

[36] J. Jaeckel, J. Redondo and A. Ringwald, Phys. Rev. Lett. 101, 131801 (2008).

[37] D. Larson et al., Astrophys. J. Suppl. 192, 16 (2011).

[38] J. R. Ellis, D. V. Nanopoulos and S. Sarkar, Nucl. Phys. B 259, 175 (1985).

[39] M. Kawasaki and T. Moroi, Astrophys. J. 452, 506 (1995).

[40] J. L. Feng, A. Rajaraman and F. Takayama, Phys. Rev. D 68, 063504 (2003).

[41] D. Lindley, Mon. Not. R. Astron. Soc. 188, 15P (1979).

[42] R. H. Cyburt, J. R. Ellis, B. D. Fields and K. A. Olive, Phys. Rev. D 67, 103521 (2003).

[43] M. Kawasaki, K. Kohri and T. Moroi, Phys. Rev. D 71, 083502 (2005).

[44] K. Jedamzik, Phys. Rev. D 74, 103509 (2006).

[45] M. Kusakabe, T. Kajino and G. J. Mathews, Phys. Rev. D 74, 023526 (2006).

[46] M. Kusakabe, T. Kajino, T. Yoshida, T. Shima, Y. Nagai and T. Kii, Phys. Rev. D 79, 123513 (2009).

[47] T. Shima et al., Phys. Rev. C 72, 044004 (2005).

[48] T. Kii, T. Shima, Y. Nagai and T. Baba, Nucl. Instrum. Meth. A 552, 329 (2005).

[49] J. R. Ellis, K. A. Olive and E. Vangioni, Phys. Lett. B 619, 30 (2005).

[50] C. Bird, K. Koopmans and M. Pospelov, Phys. Rev. D 78, 083010 (2008).

[51] M. Kusakabe et al., Phys. Rev. D 76, 121302 (2007).

[52] M. Pospelov, Phys. Rev. Lett. 98, 231301 (2007).

[53] M. Kusakabe, T. Kajino, R. N. Boyd, T. Yoshida and G. J. Mathews, Astrophys. J. 680, 846 (2008).

[54] K. Hamaguchi et al., Phys. Lett. B 650, 268 (2007).

[55] M. Kamimura, Y. Kino and E. Hiyama, Prog. Theor. Phys. 121, 1059 (2009).

[56] M. Kusakabe, T. Kajino, T. Yoshida and G. J. Mathews, Phys. Rev. D 81, 083521 (2010).

[57] T. Kajino, G. J. Mathews and G. M. Fuller, Astrophys. J. 364, 7-14 (1990).

[58] G. Mathews, T. Kajino and T. Shima, Phys. Rev. D 71, 021302 (2005).

[59] L. Randall and R. Sundrum, Phys. Rev. Lett. 83, 3370 (1999); 83, 4690 (1999).

[60] K. Ichiki, M. Yahiro, T. Kajino, M. Orito and G. J. Mathews, Phys. Rev. D 66, 043521 (2002).

[61] S. L. Dubovsky, V. A. Rubakov and P. G. Tinyakov, Phys. Rev. D62, 105011 (2000).

[62] K. Ichiki, P. M. Garnavich, T. Kajino, G. J. Mathews and M. Yahiro, Phys. Rev. D 68, 083518 (2003).

[63] K. Umezu, K. Ichiki, T. Kajino, G. J. Mathews, R. Nakamura and M. Yahiro, Phys. Rev. D 73, 063527 (2006). 